\title{
miR-126 is essential for endothelial phenotype expression during endothelial differentiation in adipose-derived stem cells
}

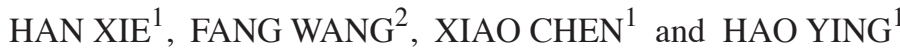 \\ ${ }^{1}$ Department of Obstetrics, Shanghai First Maternity and Infant Hospital, Tongji University School of \\ Medicine, Shanghai 201204; ${ }^{2}$ Department of Gynecology and Obstetrics, Union Hospital, Tongji Medical \\ College, Huazhong University of Science and Technology, Wuhan, Hubei 430030, P.R. China
}

Received March 13, 2016; Accepted February 27, 2017

DOI: $10.3892 / \mathrm{mmr} .2017 .7915$

\begin{abstract}
The endothelial differentiation of stem cells serves an essential role in vascular development, function and disease. Autograft adipose-derived stem cells (ADSCs) may be a novel source of cell for use in the study of angiogenesis. microRNA-126 (miR-126) has been extensively studied in endothelial cells. However, the effect of miR-126 on the endothelial differentiation of ADSCs has not been demonstrated. In the present study, it was observed that the expression of miR-126 was markedly increased during the endothelial differentiation of ADSCs in a time-dependent manner. The effect of miR-126 on ADSC differentiation was confirmed by employing up- and down-regulation strategies. The expression of endothelial markers was decreased by miR-126 inhibitor transfection during endothelial differentiation. The results of the present study suggest that miR-126 is essential for endothelial phenotype expression, and may promote the progress of endothelial differentiation in ADSCs, providing a novel strategy for modulating vascular formation and function.
\end{abstract}

\section{Introduction}

Due to the rapid development of stem cell transplantation in preclinical research and clinical trials over the last decade, stem cell replacement therapies have become modern therapeutic approaches (1). Similar to bone-marrow stromal cells, adipose-derived stem cells (ADSCs) have the ability to differentiate into various types of cell lineage, including adipogenic, osteogenic, myogenic and chondrogenic cells $(2,3)$. In addition, the yield of mesenchymal stem cells (MSCs) from

Correspondence to: Dr Hao Ying, Department of Obstetrics, Shanghai First Maternity and Infant Hospital, Tongji University School of Medicine, 2699 Gaoke West Road, Pudong, Shanghai 201204, P.R. China

E-mail: stephenying_2011@163.com

Key words: adipose-derived stem cells, cluster of differentiation 31, endothelial differentiation, endothelial nitric oxide synthase, microRNA-126, von Willebrand factor adipose tissue is $100-500$ fold that from bone marrow (4). The unique biology of autologous ADSCs, including being easily expanded, immune-privileged and capable of long-term transgene expression following multiple stages of differentiation, demonstrates their potential as a gene delivery vehicle (5-7).

The endothelial differentiation of ADSCs serves an essential role in vascular development, function and disease (8). Numerous peptide growth factors promote angiogenesis by promoting differentiation, and enhancing endothelial cell proliferation, migration and capillary network stability (9).

The knockdown of microRNA-126 (miR-126) in zebrafish in vivo resulted in the loss of vascular integrity and hemorrhage during embryonic development, and targeted deletion of miR-126 in mice caused delayed angiogenic sprouting, widespread hemorrhaging and partial embryonic lethality (10-12). These vascular abnormalities may be attributed to diminished angiogenic growth factor signaling, resulting in reduced endothelial cell differentiation, growth, sprouting and adhesion (13-16). This evidence indicates that miR-126 may be involved in multiple aspects of fundamental biological processes, including angiogenesis. However, the role of miR-126 in mediating the differentiation of ADSCs to endothelial cells has not been demonstrated.

The present study focused on the association between miR-126 and the endothelial differentiation of ADSCs.

\section{Materials and methods}

Cell growth curve and endothelial differentiation of ADSCs. ADSCs were purchased from the American Type Culture Collection (Manassas, VA, USA) and cultured in $\alpha$-Minimum Essential Medium ( $\alpha$-MEM; Gibco; Thermo Fisher Scientific, Inc., Waltham, MA, USA), supplemented with $10 \%$ fetal bovine serum (Gibco; Thermo Fisher Scientific, Inc.) at $37^{\circ} \mathrm{C}$ in $5 \% \mathrm{CO}_{2}$. Equal numbers $\left(2 \times 10^{4}\right)$ of passage $(\mathrm{p}) 1,3,6$ and 8 cells were plated and cultured. Cells images were obtained at X100 magnification using a phase-contrast inverted light microscope. Cells were harvested each day for 8 days. The total number of cells was counted in each plate using a hemocytometer. The cell growth curve was drawn by plotting the mean cell number of each plate against the culture time. For endothelial differentiation, ADSCs were cultured in Endothelial Cell Growth Medium-2 (EGM-2; Lonza, Basel, Switzerland). 
Human umbilical vein endothelial cells (HUVECs; CRL-1730; American Type Culture Collection) were cultured in F-12K medium (30-2004; American Type Culture Collection) with 10\% fetal bovine serum (30-2020; American Type Culture Collection), $0.05 \mathrm{mg} / \mathrm{ml}$ ECGS (no. 354006, BD Biosciences, Franklin Lakes, NJ, USA) and $0.1 \mathrm{mg} / \mathrm{ml}$ heparin (\#H3393; Sigma-Aldrich, Merck KGaA, Darmstadt, Germany).

Transfection. The hsa-miR-126-3p inhibitor (CGCAUUAUU ACUCACGGUACGA), hsa-miR-126-3p mimic (5-3', UCG UACCGUGAGUAAUAAUGCG; 3-5', AGCAUGGCACUC AUUAUUACGC; ), and their negative controls (NCs) (mimic NC, 5-3', UCACAACCUCCUAGAAAGAGUAGA; 3-5', AGU GUUGGAGGAUCUUUCUCAUCU; inhibitor NC, UCUACU CUUUCUAGGAGGUUGUGA; all Biomics Biotechnologies, Nantong, China) were diluted to a final concentration of $50 \mathrm{nM}$ with RNase-free $\mathrm{H}_{2} \mathrm{O}$. ADSCs were transfected respectively with these oligonucleotides using Lipofectamine 2000 (Invitrogen; Thermo Fisher Scientific, Inc.), following the manufacturer's protocol. At each time point (days 1-8), cells were harvested for further analysis. The efficiency of transfection was determined using aCy3-short interfering RNA (Cy3-siRNA) transfection control (Biomics Biotechnologies). The inhibition and overexpression efficiencies were determined by comparing with the NCs.

Immunofluorescence staining. Cells were uninduced in $\alpha$-MEM or induced in EGM-2 for 7-14 days, fixed with $4 \%$ paraformaldehyde (PFA), blocked with $10 \%$ normal goat serum (50062Z; Thermo Fisher Scientific, Inc.) for $10 \mathrm{~min}$ at room temperature and incubated with one of three primary antibodies: Rabbit anti-human cluster of differentiation (CD)31 (1:50; 11265-AP), von Willebrand factor (vWF) (1:50; 11778-1-AP) or endothelial nitric oxide synthase (eNOS) (1:50; 20116-1-AP) (all from Proteintech Group, Inc., Chicago, IL, USA) at $4^{\circ} \mathrm{C}$ overnight. Following incubation with fluorescein isothiocyanate (FITC)-conjugated goat anti-rabbit IgG (CW0114S, 1:100; CWBiotech, Beijing, China) for $3 \mathrm{~h}$ at room temperature, the cells were counterstained by incubation with DAPI (Sigma-Aldrich, Merck KGaA). Images were obtained at magnification $\mathrm{x} 200$ using a phase contrast fluorescence microscope (Olympus Corporation, Tokyo, Japan).

Reverse transcription-quantitative polymerase chain reaction $(R T-q P C R)$. Total RNA and miRNA were isolated using a miRNeasy Mini kit (Qiagen, Inc., Valencia, CA, USA). Synthesis of complementary DNA from mRNA and miRNA was performed using a PrimeScript RT reagent kit (Takara Bio, Inc., Otsu, Japan) or a miRcute miRNA First-Strand cDNA Synthesis kit (Tiangen Biotech Co., Ltd., Beijing, China), respectively. RT-qPCR analysis of mRNA and miRNA was performed using SYBR Premix Ex Taq ${ }^{\mathrm{TM}}$ (Takara Bio, Inc.) or a miRcute miRNA qPCR Detection kit (SYBR Green) (Tiangen Biotech Co., Ltd.), respectively. Amplification data was recorded using an ABI 7500 Real-Time PCR System (Applied Biosystems; Thermo Fisher Scientific, Inc.). The PCR analysis of miR-126 consisted of 38 cycles of $95^{\circ} \mathrm{C}$ for $15 \mathrm{sec}$ and $60^{\circ} \mathrm{C}$ for $1 \mathrm{~min}$, following an initial denaturation step of $95^{\circ} \mathrm{C}$ for $10 \mathrm{~min}$. The expression level of miR-126 was normalized to U6 small nuclear RNA, and the expression levels of CD31, vWF and eNOS mRNA were normalized to GAPDH. The primers used are presented in Table I. The results were subjected to melting curve analysis, and the data were analyzed using the $2^{-\Delta \Delta \mathrm{Cq}}$ method (17).

Western blot analysis. Confluent cells were removed by scraping, lysed in mammalian protein extraction reagent (Thermo Fisher Scientific, Inc.) supplemented with the protease inhibitor phenylmethylsulfonyl fluoride, and quantified using a bicinchoninic acid assay. Proteins (20 $\mu \mathrm{g} / \mathrm{lane})$ were resolved on a $10 \%$ SDS-PAGE gel and transferred onto a polyvinylidene fluoride membrane. Following blocking in 5\% non-fat dried milk and TBS with $0.1 \%$ Tween-20 (TBST) for $2 \mathrm{~h}$, the blots were incubated with a rabbit anti-human CD31 (1:500; 11265-AP), vWF (1:500; 11778-1-AP) or eNOS (1:500; 20116-1-AP) (all from ABclonal Biotech Co., Ltd., Cambridge, MA, USA) overnight at $4^{\circ} \mathrm{C}$. GAPDH $(1: 3,000$; sc-47724; Santa Cruz Biotechnology, Inc., Dallas, TX, USA) was used as an internal control. Subsequent to washing with TBST, the blots were incubated with HRP-conjugated goat anti-rabbit IgG (CW0103S, 1:2,000; CWBiotech) at room temperature for $1 \mathrm{~h}$ and visualized by enhanced chemiluminescence (GE Health care Biosciences, Pittsburgh, PA, USA). Image band area and density were estimated using Quantity One 4.6.2 (Bio-Rad Laboratories Inc., Hercules, CA, USA).

Statistical analysis. Each experiment was repeated three times independently. All data were expressed as the mean \pm standard deviation. Statistical analysis was performed using a one-way analysis of variance test and SPSS software (version 19.0; IBM SPSS, Armonk, NY, USA). $\mathrm{P}<0.05$ was considered to indicate a statistically significant difference.

\section{Results}

Morphological features of cultured ADSCs. The ADSCs attached to the culture dish surface exhibited a typical fibroblast-like morphology. The cells were maintained in culture with no sign of senescence or differentiation following repeated subculturing to p6 (Fig. 1A-C). In order to investigate whether passages affect cell growth, the number of cells was recorded every day for 8 days and compared with the cell growth curve of p1, 3, 6 and 8. Following an initial lag or stationary period (day 1), the cells expanded rapidly in a logarithmic manner until a plateau was reached (day 6). However, the ADSCs exhibited markedly more rapid growth at p3 and 6 (Fig. 1D).

Endothelial phenotype during endothelial differentiation in ADSCs. ADSCs were subjected to incubation in EGM-2 to stimulate endothelial differentiation. When cultured in medium with endothelial cell growth supplement, ADSCs expressed endothelial-specific markers, including CD31, vWF and eNOS (Fig. 2). These endothelial markers were rapidly upregulated during differentiation and remained elevated 14 days post-differentiation (data not shown).

Differential expression of miR-126 during endothelial differentiation of ADSCs. The non-induced and endothelial-induced ADSCs were harvested on days 0, 7 and 14, and analyzed for miR-126 level using RT-qPCR analysis, to determine the expression of miR-126 at the different stages of 
Table I. Primers used in the present study.

Name

Sequence $\left(5^{\prime}-3^{\prime}\right)$

$\begin{array}{ll}\text { CD31 } & \text { F, TGTATTTCAAGACCTCTGTGCACTT } \\ \text { vWF } & \text { R, TTAGCCTGAGGAATTGCTGTGTT } \\ & \text { F, TAAGTCTGAAGTAGAGGTGG } \\ \text { eNOS } & \text { R, AGAGCAGCAGGAGCACTGGT } \\ & \text { F, CAGTGTCCAACATGCTGCTGGAAATTG } \\ \text { GAPDH } & \text { R, TAAAGGAGGTCTTCTTCCTGGTGATGCC } \\ \text { miR-126-3p } & \text { F, ACCACAGTCCATGCCATCAC } \\ & \text { R, TCCACCACCCTGTTGCTGTA } \\ & \text { RT,CTCAACTGGTGTCGTGGAGTCGGCAATTCAGTTGAGCGCATTAT } \\ \text { U6 } & \text { F, ACACTCCAGCTGGGTCGTACCGTGAGTA } \\ & \text { R, CTCAACTGGTGTCGTGGAGT } \\ & \text { RT, CAAAATATGGAACGCTTC } \\ & \text { F, GTGCTCGCTTCGGCAGC } \\ & \text { R, CAAAATATGGAACGCTTC }\end{array}$

F, forward; R, reverse; RT, reverse transcription; CD31, cluster of differentiation 31; vWF, von Willebrand factor; eNOS, endothelial nitric oxide synthase; miR, microRNA.
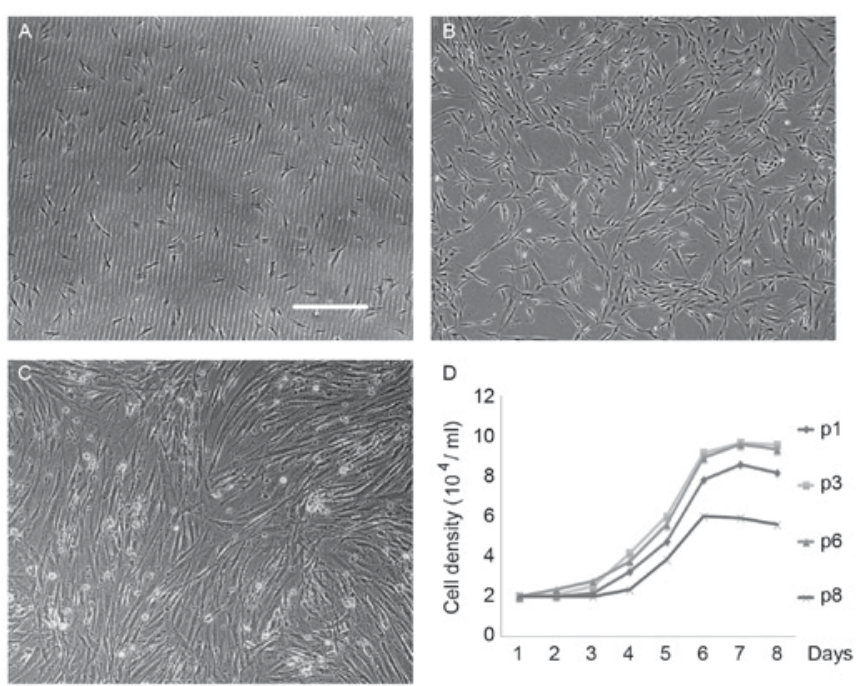

Figure 1. Biological characteristics of ADSCs. (A) The majority of cells were adherent and appeared round in morphology $24 \mathrm{~h}$ following passage Magnification, x100; scale bar, $200 \mu \mathrm{m}$. (B) After 3 days, the adherent cells in complete medium became active, proliferated quickly, formed processes, and expanded to generate small and large colonies. (C) 6 days later, the cells reached $80 \%$ confluence. Cells that attached to the culture dish surface exhibited a typical fibroblast-like morphology. (D) The growth of ADSCs was slow and limited to the first $24 \mathrm{~h}$. Representative growth curves of ADSCs in their logarithmic phase after day 3 are presented. A marked inhibition of cell growth was observed in both pland 8 compared with p3 and 6. Growth curves of $\mathrm{p} 3$ were similar to the $\mathrm{p} 6$. Cells grown at $\mathrm{p} 3$ and 6 exhibited increased growth rates, shorter lag-phase periods and higher cell concentrations, compared with $\mathrm{p} 1$ and 8 (each point represents the number of cells against time). ADSCs, adipose-derived stem cells; $p$, passage.

endothelial differentiation. It was identified that the expression of miR-126 was enriched in HUVECs and markedly increased during the endothelial differentiation of ADSCs in a time-dependent manner (Fig. 3A).

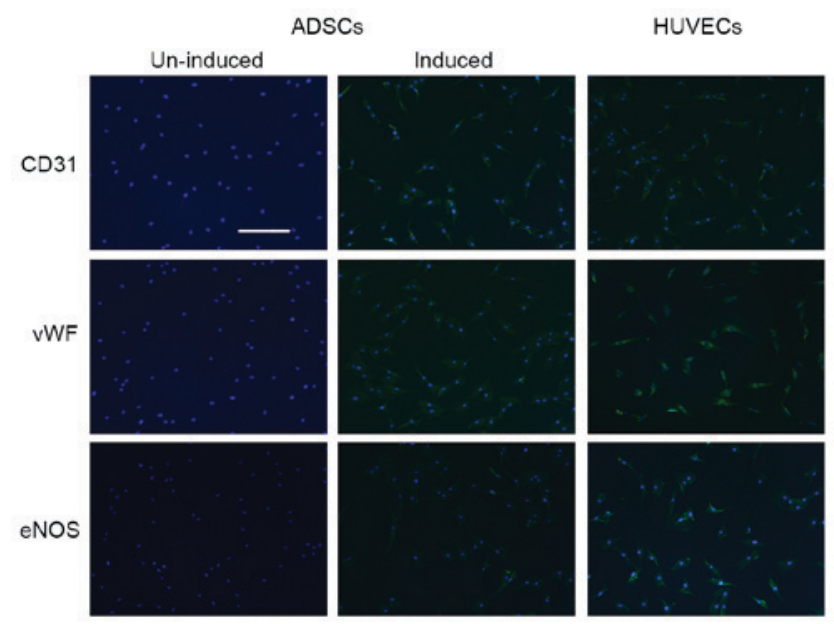

Figure 2. Endothelial differentiation of ADSCs. Immunofluorescence staining demonstrates that the expression of the three markers of endothelial differentiation CD31, vWF and eNOS, was greater in induced ADSCs compared with non-induced ADSCs. HUVECs were used as a positive control. The images exhibitthe nucleus (blue) and the expression of endothelial differentiation markers (green) at x200 magnification (scale bar, $100 \mu \mathrm{m})$. ADSCs, adipose-derived human stem cells; CD31, cluster of differentiation 31; eNOS, endothelial nitric oxide synthase; HUVECs, human umbilical vascular endothelial cells; vWF, von Willebrand factor.

Transfection of ADSCs. In order to study the effect of miR-126 in the endothelial differentiation of ADSCs, a miR-126 overexpression and inhibition model was established. After $24 \mathrm{~h}$, transfection efficiency was detected by Cy3-siRNA transfection control (siR-Rib). Following transfection with miR-126 mimic or inhibitor for 3 days, the expression of miR-126 was detected to assess the efficiency of overexpression and inhibition (Fig. 3B).

miR-126 modulates the endothelial phenotype of ADSCs. The expression of CD31, vWF and eNOS largely mirrored that of 

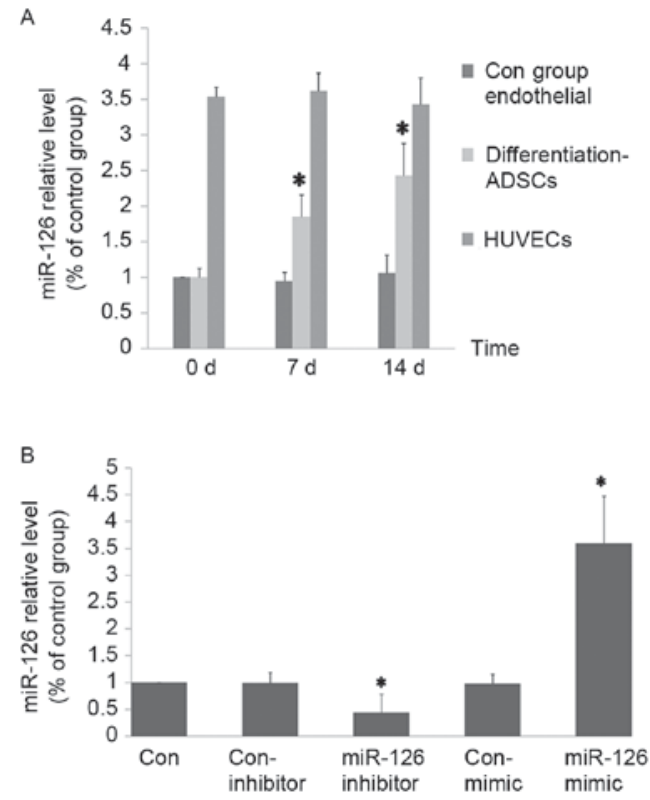

Figure 3. miR-126 level in ADSCs. (A) ADSCs expressed an increased level of miR-126 during endothelial differentiation. RT-qPCR analysis demonstrated that the expression level of miR-126 ADSCs was low. miR-126 was induced at day 7 of endothelialization and further increased at day 14 . The miR-126 level in HUVECs was used as a positive control. (B) The expression of miR-126 in transfected ADSCs was confirmed by RT-qPCR. miR-126 was expressed in all the groups and showed a greater increase in miR-126 mimic-transfected cells, whereas miR-126 expression was substantially decreased by treatment with a miR-126 inhibitor. RT-qPCR analysis revealed no significant difference in miR-126 expression between mimic control and inhibitor control groups. " $\mathrm{P}<0.05$ vs. respective Con. ADSCs, adipose-derived stem cells; Con, control; RT-qPCR, quantitative polymerase chain reaction; miR, microRNA; HUVECs, human umbilical vascular endothelial cells.

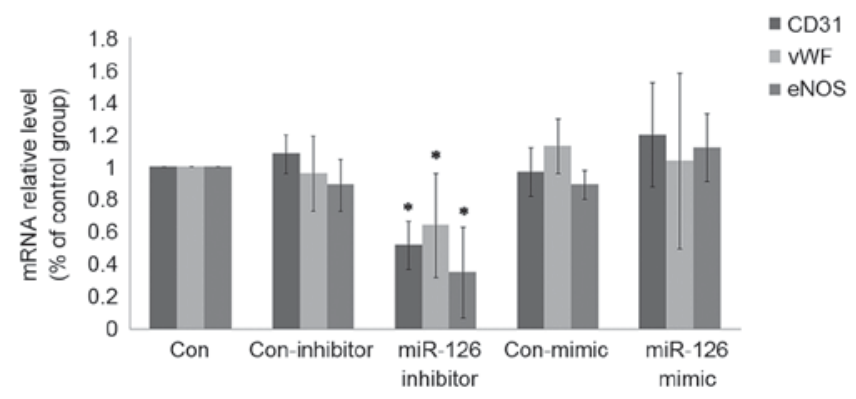

Figure 4. miR-126 modulates endothelial phenotype at themiR level. The expression of endothelial markers was decreased by miR-126 inhibitor transfection during endothelial differentiation as demonstrated by RT-qPCR analysis. Endothelial marker expression was not affected by miR-126 overexpression. ${ }^{*} \mathrm{P}<0.05$ vs. ADSCs transfected with Con inhibitor. ADSCs, adipose-derived stem cells; CD31, cluster of differentiation 31; Con, control; eNOS, endothelial nitric oxide synthase; RT-qPCR, quantitative polymerase chain reaction; miR, microRNA; vWF, von Willebrand factor.

endothelial markers during endothelialization. The expression of these markers was decreased by miR-126 inhibitor transfection during endothelial differentiation at the mRNA (Fig. 4) and protein level (Fig. 5). In addition, ADSCs with miR-126 overexpression were generated through transfection of a miR-126 mimic. It was identified that the expression of endothelial markers was not notably affected by miR-126 overexpression.

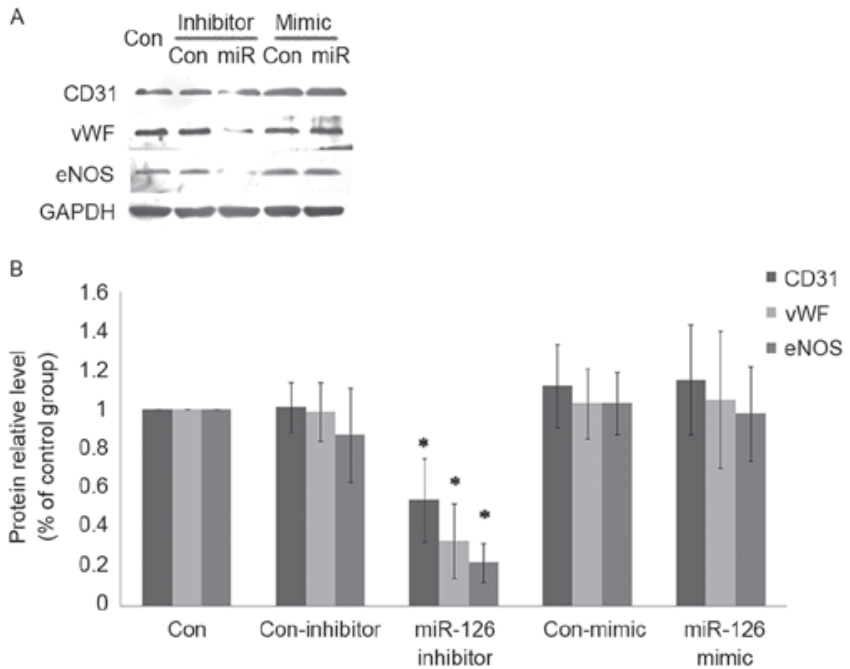

Figure 5. miR-126 modulates endothelial phenotype at the protein level. (A) Protein expression of endothelial markers CD31, vWF and eNOS. GAPDH was used as an internal control. (B) Expression of endothelial markers was decreased by miR-126 inhibitor transfection during endothelial differentiation as demonstrated by western blot analysis. The expression of endothelial markers was not affected by miR-126 overexpression. " $\mathrm{P}<0.05$ vs. ADSCs transfected with Con inhibitor. ADSCs, adipose-derived stem cells; CD31, cluster of differentiation 31; Con, control; vWF, von Willebrand factor; eNOS, endothelial nitric oxide synthase; miR, microRNA.

\section{Discussion}

ADSCs may be easily harvested from lipoaspirate and the extraction maybe less invasive and less expensive compared with extraction from bone marrow. Additionally, ADSCs have a significantly shorter doubling time when expanded in vitro (18). In the present study, ADSCs of $\mathrm{p} 3-6$ were used for the experiments and it was observed that the majority of the cells exhibited typical fibroblastoid morphology, expressed MSC surface markers, and demonstrated the capability for multipotency (data not shown). These intrinsic characteristics and advantages make ADSCs an ideal stem cell source for the present study and future cell-based tissue engineering and therapies $(19,20)$.

In the present study, expression analyses revealed an abundant level of miR-126 in HUVECs and identified miR-126 as one of the miRs known to be specifically expressed in the endothelial cell lineage. Expression of miR-126 gradually increased during ADSC endothelial differentiation, and expression was marked when endothelial markers were expressed. Endothelial differentiation markers (CD31, vWF and eNOS) were used to evaluate the differentiation capability of ADSCs. The results of the present study suggest that miR-126 may be involved in the directional differentiation of ADSCs into vascular endothelial cells, and may promote the progress of the differentiation.

Due to the high expression of miR-126 in HUVECs, and the increased expression during the endothelial differentiation of ADSCs, the potential role of this miR in the regulation of differentiation towards the endothelial lineage was investigated. In order to confirm the role of miR-126 in the endothelial differentiation of ADSCs, down- and up-regulation of miR-126 expression was achieved through transfection withmiR-126 inhibitor and mimic, respectively. As a result 
of miR-126 inhibition in ADSCs undergoing the endothelial differentiation, diminished mRNA expression of endothelial cell markers was observed. These results demonstrated that the downregulation of miR-126 may exhibit a negative effect on endothelial differentiation. By contrast, the overexpression of miR-126 did not improve the expression levels of endothelial markers. The results of the present study suggest that while miR-126 is enriched in vascular endothelial cells, and is essential to endothelial differentiation, it is not sufficient to promote the differentiation of ADSCs towards an endothelial phenotype.

Previous research has demonstrated that miR-126 serves an essential role in stem cell differentiation (21) and angiogenesis. However, the mechanism remains unknown and there is a lack of consistency between studies. It is difficult to connect the physiological functions of miR-126 in vasculature to its functions in tumor. It has been established that angiogenesis represents one of the key features in the pathogenesis of cancer (22), but little is known about the role of miR-126 in tumor neoangiogenesis. The present study suggests that miR-126 may have a supportive role in the progression of cancer, which maybe mediated by the promotion of blood vessel growth. By contrast, it was reported that the downregulation of miR-126 increased the activity of vascular endothelial growth factor-A in lung and breast cancer (23-25). miR-126 is regarded as a putative tumor suppressor due to its potential role in anti-angiogenesis in cancer (26). Consequently, miR-126 may function differently in stromal cells compared with tumor cells. The effect and mechanism of miR-126 on angiogenesis in different cells or tissue requires further research, to compare the effects of miR-126 in tumor cells with the functions of miR-126 in tumor vasculature and the surrounding vessels of the tumor periphery.

\section{Acknowledgements}

The present study was supported by a grant from the National Natural Science Foundation of China (grant no. 81200443).

\section{References}

1. Trounson A and McDonald C: Stem Cell Therapies in Clinical Trials: Progress and Challenges. Cell Stem Cell 17: 11-22. 2015.

2. Yang Y, Chen XH, Li FG, Chen YX, Gu LQ, Zhu JK and Li P: In vitro induction of human adipose-derived stem cells into lymphatic endothelial-like cells. Cell Reprogram 17: 69-76. 2015.

3. Hu F, Wang X, Liang G, Lv L, Zhu Y, Sun B and Xiao Z: Effects of epidermal growth factor and basic fibroblast growth factor on the proliferation and osteogenic and neural differentiation of adipose-derived stem cells. Cell Reprogram 15: 224-232. 2013.

4. De Ugarte DA, Morizono K, Elbarbary A, Alfonso Z, Zuk PA, Zhu M, Dragoo JL, Ashjian P, Thomas B, Benhaim P, et al: Comparison of multi-lineage cells from human adipose tissue and bone marrow. Cells Tissues Organs 174: 101-109. 2003.

5. Ikegame Y, Yamashita K, Hayashi S, Mizuno H, Tawada M, You F, Yamada K, Tanaka Y, Egashira Y, Nakashima S, et al: Comparison of mesenchymal stem cells from adipose tissue and bone marrow for ischemic stroke therapy. Cytotherapy 13: 675-685. 2011.

6. Locke M, Feisst V and Dunbar PR: Concise review: Human adipose-derived stem cells: Separating promise from clinical need. Stem Cells 29: 404-411. 2011.
7. Khan WS, Adesida AB, Tew SR, Longo UG and Hardingham TE: Fat pad-derived mesenchymal stem cells as a potential source for cell-based adipose tissue repair strategies. Cell Prolif 45: 111-120. 2012.

8. Fraser JK, Wulur I and Alfonso Z and Hedrick MH: Fat tissue: An underappreciated source of stem cells for biotechnology. Trends Biotechnol 24: 150-154. 2006.

9. Park IS, Rhie JW and Kim SH: A novel three-dimensional adipose-derived stem cell cluster for vascular regeneration in ischemic tissue. Cytotherapy 16: 508-522. 2014.

10. Fish JE, Santoro MM, Morton SU, Yu S, Yeh RF, Wythe JD, Ivey KN, Bruneau BG, Stainier DY and Srivastava D: miR-126 regulates angiogenic signaling and vascular integrity. Dev Cell 15: 272-284. 2008 .

11. Wang S, Aurora AB, Johnson BA, Qi X, McAnally J, Hill JA, Richardson JA, Bassel-Duby R and Olson EN: The endothelial-specific microRNA miR-126 governs vascular integrity and angiogenesis. Dev Cell 15: 261-271. 2008.

12. Kuhnert F, Mancuso MR, Hampton J, Stankunas K, Asano T, Chen CZ and Kuo CJ: Attribution of vascular phenotypes of the murine Egfl7 locus to the microRNA miR-126. Development 135: 3989-3993. 2008.

13. Sasahira T, Kurihara M, Bhawal UK, Ueda N, Shimomoto T, Yamamoto K, Kirita T and Kuniyasu H: Downregulation of miR-126 induces angiogenesis and lymphangiogenesis by activation of VEGF-A in oral cancer. Br J Cancer 107: 700-706. 2012.

14. Nowak WN, Florczyk U, Józkowicz A and Dulak J: Role of microRNA in endothelial cells-regulation of differentiation and angiogenesis. Postepy Biochem 59: 405-414. 2013 (In Polish)

15. Yan T, Liu Y, Cui K, Hu B, Wang F and Zou L: MicroRNA-126 regulates EPCs function: Implications for a role of miR-126 in preeclampsia. J Cell Biochem 114: 2148-2159. 2013.

16. Meister J and Schmidt MH: miR-126 and miR-126*: New players in cancer. ScientificWorldJournal 10: 2090-2100. 2010.

17. Livak KJ and Schmittgen TD: Analysis of relative gene expression data using real-time quantitative PCR and the 2(-Delta Delta C(T)) method. Methods 25: 402-408, 2001.

18. Ikegame Y, Yamashita K, Hayashi S, Mizuno H, Tawada M, You F, Yamada K, Tanaka Y, Egashira Y, Nakashima S, et al: Comparison of mesenchymal stem cells from adiposetissue and bonemarrow for ischemicstroke therapy. Cytotherapy 13: 675-685. 2011.

19. Nakagami H, Maeda K, Morishita R, Iguchi S, Nishikawa T, Takami Y, Kikuchi Y, Saito Y, Tamai K, Ogihara T and Kaneda Y: Novel autologous cell therapy in ischemic limb disease through growth factor secretion by cultured adipose tissue-derived stromal cells. Arterioscler Thromb Vasc Biol 25: 2542-2547. 2005.

20. Su SJ, Yeh YT, Su SH, Chang KL, Shyu HW, Chen KM and Yeh H: Biochanin a promotes osteogenic but inhibits adipogenic differentiation: Evidence with primary adipose-derived stem cells. Evid Based Complement Alternat Med 2013: 846039. 2013.

21. Wu Z, Yin H, Liu T, Yan W, Li Z, Chen J, Chen H, Wang T, Jiang Z, Zhou W and Xiao J: MiR-126-5p regulates osteoclast differentiation and bone resorption in giant cell tumor through inhibition of MMP-13. Biochem Biophys Res Commun 443: 944-949. 2014.

22. El-Kenawi AE and El-Remessy AB: Angiogenesis inhibitors in cancer therapy: Mechanistic perspective on classification and treatment rationales. Br J Pharmacol 170: 712-729. 2013.

23. Liu B, Peng XC, Zheng XL, Wang J and Qin YW: MiR-126 restoration down-regulate VEGF and inhibit the growth of lung cancer cell lines in vitro and in vivo. Lung Cancer 66: 169-175. 2009.

24. Zhu X, Li H, Long L, Hui L, Chen H, Wang X, Shen H and $\mathrm{Xu} \mathrm{W}$ : miR-126 enhances the sensitivity of non-small cell lung cancer cells to anticancer agents by targeting vascular endothelial growth factor A. Acta Biochim Biophys Sin (Shanghai) 44: 519-526. 2012.

25. Zhu N, Zhang D, Xie H, Zhou Z, Chen H, Hu T, Bai Y, Shen Y, Yuan W, Jing Q and Qin Y: Endothelial-specific intron-derived miR-126 is down-regulated in human breast cancer and targets both VEGFA and PIK3R2. Mol Cell Biochem 351: 157-164. 2011.

26. Feng R, Chen X, Yu Y, Su L, Yu B, Li J, Cai Q, Yan M, Liu B and Zhu Z: miR-126 functions as a tumour suppressor in human gastric cancer. Cancer Lett 298: 50-63. 2010. 